\title{
Diabetes is the Leading Cause of ESRD in Haemodialysis Patients
}

\author{
TANIA MAHBUB,${ }^{1}$ MD. NIZAM UDDIN CHOWDHURY, ${ }^{1}$ FERDOUS JAHAN, ${ }^{1}$ SAKIBJJAMAN AREFEEN, ${ }^{2}$ \\ MOTLEBUR RAHMAN, ${ }^{3}$ MS SARKER $^{1}$
}

\begin{abstract}
Diabetes is one of the leading causes of ESRD and the no of diabetes patients is increasing day by day.In the developed world it is the first and foremost cause of renal failure. However in the developing countries it has been considered as second most common cause after GN.This study was a cross sectional study conducted during Oct 2010 to Sept 20II, in the dept of Nephrology, Dhaka Medical College and Hospital to determine the cause of renal failure with specific object to find out the leading cause of ESRD among the Haemodialysis patients. This study found Diabetes as the leading cause of ESRD accounting $44.1 \%$ of whole population.
\end{abstract}

Keywords: Diabetes, ESRD, Haemodialysis

\section{Introduction}

Diabetes is the most common cause of kidney failure, and nearly 180,000 people are living with kidney failure as a result of diabetes and accounting for nearly 44 percent of new cases. ${ }^{1}$ Even when diabetes is controlled, the disease can lead to CKD and kidney failure. Nearly 24 million people in the United States have diabetes, each year in the United States, more than 100,000 people are diagnosed with kidney failure. ${ }^{2}$ Diabetic Nephropathy (DN) is the single most important cause of end stage renal disease (ESRD) in Europe also. ${ }^{6}$ Currently India has the largest number of diabetic individual-even more than USA and China. It was estimated that there were 30million diabetes in India and the no will increase to 80 million by 2030.People with kidney failure undergo either dialysis, or transplantation. Most U.S. citizens who develop kidney failure are eligible for federally funded care. In 2005, care for patients with kidney failure cost the United States nearly $\$ 32$ billion. ${ }^{1}$

There is no cure for diabetic nephropathy, and treatment is lifelong. Around 20 to 30 per cent of people with diabetes develop diabetic nephropathy, although not all of these will progress to kidney failure. A person with diabetes is susceptible to nephropathy whether they use insulin or not. The risk is related to the length of time the person has diabetes. ${ }^{3}$

People with diabetes are also at risk of other kidney problems, renal artery stenosis or renovascular disease. It is an important cause of morbidity and mortality and It is the leading cause of premature death in young diabetic patients. ${ }^{4}$ It is now the most common causes of end stage renal failure in developed countries. As it is found with other microvascular and macro vascular complications, management is frequently difficult and the benefits of prevention are substantial. ${ }^{3}$ Diabetic nephropathy is one of the most serious complications in diabetes and proteinuria is associated with an enormously excess mortality and high incidence of severe non-renal complication. According to friedman ${ }^{3}$, diabetes is the genetic disease that ranks first as a cause of end stage renal disease in adults. As increasing numbers of diabetic patients survive the earlier hazards of the disease, more will live long enough to develop renal failure that robs the sufferer and society of years of vigorous and productive activity and put a heavy burden upon the health services that provides expensive forms of the therapy. Until prevention of diabetes or diabetic complication is possible, diabetic nephropathy will occur and diabetic patients will need active treatment for their uremia.

In Bangladesh diabetic nephropathy was the second common cause of ESRD after Glomerulonephritis (45\%), accounting for about $34 \%$ of cases. ${ }^{6,10}$ Thus it is important to re evaluate diabetes as a cause of ESRD.

\section{Objective}

To detect the cause of renal failure in haemodialysis patients with a specific object to find put the leading cause of renal failure.

\section{Method}

This was a cross sectional study done in the department of nephrology during Jan 2010 to Sept 2010. All the patients of Haemodialysis unit were invited to participate in the study .Informed written concent was obtained from each patients who were willing to participate in the study . The ESRD patients who were getting dialysis were included in the study. 118 patients were included in the study. ESRD was defined by permanent and irreversible loss of renal function requiring renal replacement therapy. The cause of renal failure was determined by detailed history, through clinical examination and investigations. The causes of renal diseases were defined by following criteria. Diabetis Mellitus (DM) [Long history of diabetes, Documentation of diabetic retinopathy, Presence other diabetic complications, Documentation of insulin and oral hypoglycaemic use. Glomerulonephritis (GN)

1. Dept. of Nephrology, Dhaka Medical College, Dhaka

2. Assistant Registrar, Dept of Nephrology, Dhaka Medical College, Dhaka

3. Consultant, Dept of Medicine, Dhaka Medical College, Dhaka

Correspondence : Dr. Tania Mahbub, Dept. of Nephrology, Dhaka Medical College, Dhaka 
was diagnosed by Suggestive History, Previous documentation (biopsy and treatment history).Hypertension (HTN) was addressed by Long history of HTN, History or documentation of antihypertensive use ,Absence of other disease criteria. Obstructive Nephropathy Suggestive history, Investigation findings of stone, stricture, hydronephrosis and from previous documentation. Autosomal Dominant Polycystic Kidney Disease (ADPKD): Family history, Examination: Bilaterally palpable kidney, Investigation: Multiple cysts in both kidney as per ravine criteria. Unknown Cause of renal failure could not be defined as per above mentioned criteria.

\section{Results:}

Out of 118 patients age 18-75yrs.Gender male 70 and female 48with male female ratio 2.5:1.the average duration of haemodialysis was 9 months. DM was found $44.1 \%$. GN was found $29.7 \%$. Hypertension was found $7.6 \%$. Both the ADPKD and obstructive nephropathy were found $4.2 \%$ and $10.2 \%$ were due to unknown aetiology. Among the diabetic patients the average duration of diabetes was 10.6 years with a range 4.5 to 21 years. All most all patients received antidiabetic agent during the course of their illness with oral $43 \%$ and $76 \%$ (multiple responses) insulin. At the stage of ESRD 58\% do not require anti diabetic agents. Diabetic retinopathy was found in $48.4 \%$ population, Peripheral neuropathy $31 \%$, diabetic foot $8 \%$,coronary artery disease $62 \%$, Stroke $12 \%$, peripheral vascular disease $17 \%$.

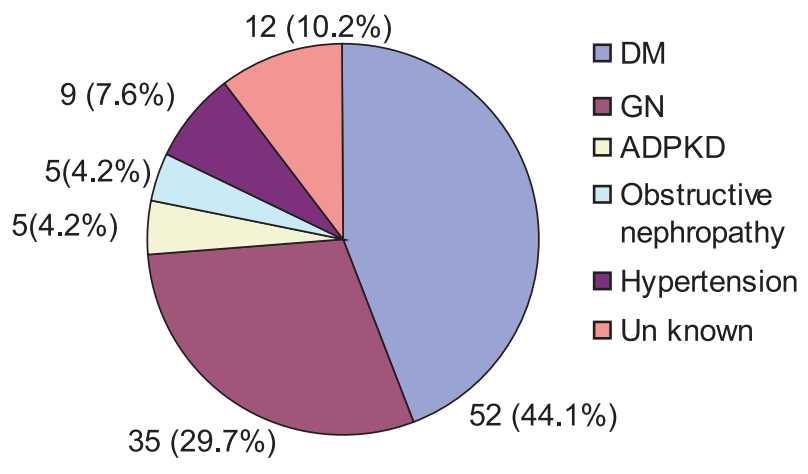

Fig.-1: Pie diagram showing the cause of ESRD of the study patients.

\section{Discussion:}

In the current study diabetes was the leading cause of renal failure and the second common cause was GN. Which was almost similar to other studies. According to (USRDS2007) DN 43.8\%, HTN 26.8\%,GN 7.6\%, Cystic disease 2.3\%, Urologic disease $2 \%$ and others $17.5 \%{ }^{1}$ A changing trend of leading cause of ESRD might be due to increase life expectancy in diabetes patients due to active management causing them to suffer from diabetes for prolong duration thus they are succeptible to more diabetic related complications .moreover successful management of glomelulonephritis due to early detection and treatment caused decreased number of ESRD due to Glomerulonephritis origin.

Though the HTN was the second cause of ESRD according to USRDS, in our study GN was next to DN. Progression to advanced nephropathy takes place 4 to 6 years of macroalbuminuria which reaches to end stage renal disease (ESRD) requiring renal replacement therapy (RRT) in next 1 to 3 years. The cost of any form of RRT (either transplantation or dialysis) is very high and in the existing circumstances, less than 5\% ESRD patients can avail this treatment and more than 95\% dies without treatment in Bangladesh. ${ }^{6}$ Furthermore, ESRD from diabetic nephropathy once on RRT, mortality is 1.5 to 2.5 times higher than non-diabetic patients, mostly due to increased cardiovascular mortality. ${ }^{9}$

Less than $20 \%$ of diabetic patients survive on dialysis after 5 years and even on RRT, the prognosis of ESRD due to diabetic nephropathy is worse than any other cause of ESRD. There has been steady improvement in the outcome of these patients over past decade, but the statistics remain dismal, which highlights the urgency to treat this particular group of patients. That is why, it is of utmost importance to prevent or at least delay the progression to ESRD from diabetic nephropathy. ${ }^{8}$

\section{Conclusion:}

Diabetes is the leading cause of renal failure in haemodialysis patients accounting $44.1 \%$ of ESRD population, like other developed countries but the disease can be successfully controlled by different measures. We should be more cautious in dealing these group of population to prevent progression in to ESRD.

\section{Limitation:}

This was a single centre study and it was conducted over a small period and the sample size was small.

\section{Recommendation:}

A large multi center study should be concucted to verify the presence of diabetes as a leading cause of ESRD and rigorious control of diabetic and care should launched from the early stage of diabetes to prevent further progression.

\section{Conflict of Interest : None}

\section{References:}

1. United States Renal Data System. USRDS 2007 Annual Data Report. Bethesda, MD: National Institute of Diabetes and Digestive and Kidney Diseases, National Institutes of Health, U.S. Department of Health and Human Services; 2007. 
2. National Institute of Diabetes and Digestive and Kidney Diseases. National Diabetes Statistics, 2007. Bethesda, MD: National Institutes of Health, U.S. Department of Health and Human Services, 2008.

3. Frer, M. Fiusher, Diabetes Millitus. David son's principles and practice of Medicine 21 ed.Churchill Livingstone Elsevier 2010; 21: 829 .

4. Parveen K, Michael C. Diabetes Mallitus and other disorders of metabolism. Kumar and clark clinical Medicine 7 ed, Elsevier 2009; 11: 1126.

5. Sumir KS, Abdur RK,Diabetic nephropathy: A short review. Bangladesh renal journal. 1989; 3(1) : 23-30.

6. Jamal UA. Diabetic Nephropathy Review Article. BIRDEM. Dhaka. 2007;33-68
7. Alvin C. Powers. Diabetes Mellitus. In: Braunwald E, Fauci A, Kasper D, Hauser SL, Longo DL and Jameson JL, editors. Harrison's Principles of Internal Medicine. 11 ed. New York: McGraw — Hill. 2001: 212-6.

8. Nelson RG, Bennett PH, Beck GJ, Tan M, Knowler WS and Myers BD. Development and progression of renal disease in Pima Indians with non ins diabetes mellitus. N EngI J Med 1996; 335: 1636-42.

9. Gall MM, Nielsen FS, Smidt UM and Parving HH. The course of kidney function in type 2 (non-insulin-dependent) diabetic patients with diabetic nephropathy. Diabetologia 1993; 36: 1071-78.

10. Reports of President, $9^{\text {th }}$ Conference of NUTS of SAARC Country, December, 2011. 